\title{
Changes in cardiac mitochondrial aldehyde dehydrogenase 2 activity in relation to oxidative stress and inflammatory injury in diabetic rats
}

\author{
HONG-JU WANG ${ }^{1 *}$, PIN-FANG KANG ${ }^{1-3^{*}}$, WEN-JUAN WU ${ }^{4 *}$, YANG TANG ${ }^{1}$, QIANG-QIANG PAN ${ }^{1-3}$, \\ HONG-WEI YE ${ }^{2,3}$, BI TANG ${ }^{1}$, ZHENG-HONG LI ${ }^{2}$ and QIN GAO $^{2,3}$ \\ ${ }^{1}$ Department of Cardiovascular Disease, The First Affiliated Hospital of Bengbu Medical College, Anhui 233004; \\ ${ }^{2}$ Department of Physiology, ${ }^{3}$ Anhui Key Laboratory of Tissue Transplantation and ${ }^{4}$ Department of Biochemistry \\ and Molecular Biology, Bengbu Medical College, Anhui 233030, P.R. China
}

Received January 27, 2013; Accepted June 4, 2013

DOI: $10.3892 / \mathrm{mmr} .2013 .1524$

\begin{abstract}
The aim of the present study was to determine the changes in mitochondrial aldehyde dehydrogenase 2 (ALDH2) activity in relation to oxidative stress and inflammatory injury in different stages of diabetes mellitus (DM) in rats and to investigate the related mechanisms. DM in Sprague-Dawley (SD) rats was induced by a single intraperitoneal injection of $55 \mathrm{mg} / \mathrm{kg}$ streptozotocin (STZ). The rats were randomly allocated into a control group, as well as into DM4w, DM8w and DM12w groups containing DM rats 4, 8 and 12 weeks after DM induction, respectively. Ventricular hemodynamic parameters were recorded; fasting blood glucose (FBG) and glycosylated hemoglobin (HbAlc) levels were determined using an automatic biochemistry analyzer; plasma interleukin (IL)-1, IL-4 and cardiac 4-hydroxynon-2-enal (4-HNE) levels were determined using enzyme-linked immunosorbent assay (ELISA), and cardiac ALDH2 activity was measured. The mRNA expression levels of Bax and Bcl-2 of the left anterior myocardium were detected by reverse transcriptase-polymerase chain reaction (RT-PCR). FBG and HbAlc levels were increased in the DM groups compared to the control group. FBG levels were not significantly different among the DM4w, DM8w and DM12w groups, while HbA1c levels were increased with the progression of diabetes. The left ventricular developed pressure (LVDP), heart rate (HR) and rate-pressure product (RPP) were decreased, plasma IL-1 levels were increased, while IL-4 levels were decreased in the DM groups compared to the control group. Additionally, cardiac 4-HNE levels were
\end{abstract}

Correspondence to: Dr Qin Gao, Department of Physiology, Bengbu Medical College, 2600 Dong Hai Avenue, Bengbu, Anhui 233030, P.R. China

E-mail: bbmcgq@126.com

*Contributed equally

Key words: diabetes, aldehyde dehydrogenase 2, 4-hydroxynon-2enal, inflammatory injury, apoptosis increased, and ALDH2 activity was decreased in the DM groups compared to the control group. Bax mRNA levels were increased, Bcl-2 mRNA levels were decreased, and Bcl-2/Bax mRNA ratios were decreased in the DM groups compared to the control group. Moreover, LVDP, HR, RPP, IL-4, ALDH2 activity and Bcl-2/Bax mRNA ratios were further reduced, while 4-HNE and IL-1 levels were increased with the progression of diabetes. In conclusion, our results indicated that cardiac ALDH2 activity was further decreased with the progression of diabetes, which might be related to the increase of oxidative stress, inflammatory injury and the occurrence of apoptosis.

\section{Introduction}

Diabetes mellitus (DM) is a common metabolic disorder with acute and chronic complications, that affects survival and quality of life of the patient (1-3). Diabetic cardiomyopathy is one of the major causes of morbidity and mortality in diabetic patients, and may cause cardiac glucose metabolism disorders, calcium overload and cardiac fibrosis, which induce cardiac structural and functional abnormalities. However, the mechanisms underlying diabetic cardiomyopathy remain to be elucidated.

Long-term high blood glucose levels may lead to complications in cardiac glucose uptake and utilization, affect the metabolism of cardiac myocytes, and ultimately lead to myocardial systolic dysfunction (4). Hyperglycemia may also increase the overproduction of reactive oxygen species (ROS), including superoxide anion, hydroxyl radicals and hydrogen peroxide $\left(\mathrm{H}_{2} \mathrm{O}_{2}\right)$. Oxidative stress caused by these free radicals has been suggested to play a crucial role in the pathogenesis of diabetic cardiomyopathy $(5,6)$.

Mitochondrial aldehyde dehydrogenase 2 (ALDH2) is one of the key enzymes in alcohol metabolism that catalyzes the conversion of aldehyde to acetic acid (7). ALDH2 plays a crucial metabolic role in the detoxification and oxidation of aldehydes, such as 4-hydroxynon-2-enal (4-HNE). 4-HNE is a highly cytotoxic aldehyde generated during oxidative stress as a result of lipid peroxidation (8-10). ALDH2 has been found to attenuate ethanol-induced myocardial dysfunction 
by regulating Akt and AMPK signaling pathways (11-13). The activation of ALDH2 has been shown to lead to cardiac protection against ischemia and reperfusion injury (14-16). A previous study also indicated that inhibition of ALDH2 by oxidative stress leads to cardiac dysfunction in diabetes mellitus (17). Nevertheless, the association between ALDH2 and myocardial injury in diabetic cardiomyopathy mediated by oxidative stress has yet to be fully elucidated.

Inflammatory injury is involved in the pathology and complications of diabetes. Hyperglycemia and oxidative stress cause inflammatory injury, eventually inducing cell death. Interleukin (IL)-1 is a pro-inflammatory cytokine that regulates inflammatory reaction $(18,19)$ and IL-4 is a cytokine with anti-inflammatory properties.

Consequently, we hypothesized that cardiac ALDH2 activity is altered with DM progression, and that this is associated with inflammatory injury. To test this hypothesis, we determined the changes in ALDH2 activity in relation to oxidative stress and inflammatory injury in different stages of DM in rats.

\section{Materials and methods}

Animals. Male Sprague-Dawley (SD) rats of 200-250 g were purchased from the Animal Center of Bengbu Medical College (Bengbu, China). The rats were fed normal chow and had free access to water. The rats were maintained at a constant temperature of $21 \pm 1^{\circ} \mathrm{C}$ on a fixed 12-h light/dark cycle. All the animal procedures were in accordance with the United States National Institutes of Health Guide and were approved by the Animal Use and Care Committee of Bengbu Medical College.

Chemicals and reagents. Streptozotocin (STZ) was purchased from Sigma (St. Louis, MO, USA). IL-1, IL-4 and 4-HNE kits were purchased from R\&D Systems (Minneapolis, MN, USA). The ALDH2 kit was purchased from Genmed Scientific Inc. (Shanghai, China). The primers used were: Bax 5'-GGA TCG AGC AGA GAG GAT GG-3' and 5'-GCT CAT TGC CGA TAG TGA TGA CT-3', with an amplified fragment length of 464 bp; Bcl-2: 5'-CTG GTG GAC AAC ATC GCT CTG-3', 5'-GGT CTG CTG ACC TCA CTT GTG-3', with an amplified fragment length of $227 \mathrm{bp} ; \beta$-actin: 5'-GAT GGT GGG TAT GGG TCA GAA GGA C-3' and 5'-GCT CAT TGC CGA TAG TGA TGA CT-3', with an amplified fragment length of 630 bp. Any additional chemicals were of the highest purity available.

Induction of diabetes and experimental protocol. Diabetes was induced in overnight fasted rats by the administration of a single intraperitoneal (i.p.) injection of $55 \mathrm{mg} / \mathrm{kg} \mathrm{STZ} \mathrm{freshly}$ dissolved in $0.1 \mathrm{~mol} / 1$ sodium citrate buffer $(\mathrm{pH} 4.5)$. The rats in the control group were intraperitoneally injected with the same dose of sodium citrate buffer alone. The rats with a fasting blood glucose (FBG) level of $>16.7 \mathrm{mmol} / 172 \mathrm{~h}$ after the injection were considered diabetic.

The rats were randomly allocated into a control group, as well as into DM4w, DM8w and DM12w groups containing DM rats 4, 8 and 12 weeks after DM induction, respectively ( $\mathrm{n}=6 \mathrm{rats} /$ group).

Isolated perfused heart preparation. After the rats were anesthetized (chloral hydrate, $4 \mathrm{~g} / \mathrm{kg}$, i.p.), the hearts were rapidly excised, placed in ice-cold Krebs-Henseleit (K-H) buffer, mounted on a Langendorff apparatus, and perfused at $37^{\circ} \mathrm{C}$ with $\mathrm{K}-\mathrm{H}$ buffer. The buffer was equilibrated with $95 \% \mathrm{O}_{2} / 5 \%$ $\mathrm{CO}_{2}$ ( $\mathrm{pH} 7.4$ ), and was composed of (mmol/1): $\mathrm{NaCl} 118.0$, $\mathrm{KCl} 4.7, \mathrm{CaCl}_{2}$ 1.25, $\mathrm{KH}_{2} \mathrm{PO}_{4} 1.2, \mathrm{NaHCO}_{3} 25.0$ and glucose 11.0. A latex, fluid-filled balloon was introduced into the left ventricle through the left atrial appendage and the balloon catheter was connected to a pressure transducer connected to a data acquisition system (MedLab, Nanjing, China) to assess contractile function. The left ventricular end diastolic pressure (LVEDP) was adjusted to 4-8 $\mathrm{mmHg}$. The cardiac parameters including heart rate (HR), left ventricular developed pressure (LVDP, difference between left ventricular end systolic pressure and end diastolic pressure) and rate-pressure product $(\mathrm{RPP}=\mathrm{LVDP} \times \mathrm{HR})$ were continuously monitored.

Detection of blood glucose and glycosylated hemoglobin (HbAlc) levels. FBG and HbA1c levels were measured every 4 weeks following the STZ injection.

Detection of plasma IL-1 and IL-4 levels. After the animals were anesthetized, $2 \mathrm{ml}$ of blood was drawn from the abdominal aorta, and plasma IL-1 and IL-4 levels were measured using commercially available kits according to the manufacturer's instructions.

Detection of cardiac 4-HNE levels. At the end of the experimental period, $0.1 \mathrm{~g}$ of heart tissue was homogenized in ice-cold phosphate-buffered saline (PBS). 4-HNE was measured by commercially available kits according to the manufacturer's instructions.

Detection of Bax and Bcl-2 mRNA levels by reverse transcriptase-polymerace chain reaction (RT-PCR). RT-PCR was used to detect the mRNA levels of Bax and Bcl-2 in the hearts of rats. Briefly, total RNA was extracted using TRIzol reagent according to the manufacturer's instructions. Total RNA $(2 \mu \mathrm{g})$ were reverse transcribed to $\mathrm{CDNA}$, and PCR was performed using a routine method. PCR products were analyzed on $1 \%$ agarose gel. Densitometric analysis results of Bax and Bcl-2 genes were compared to the corresponding $\beta$-actin levels in order to account for loading differences.

Detection of ALDH2 activity. ALDH2 activity was measured using commercially available kits according to the manufacturer's instructions.

Statistical analysis. Values were expressed as the means \pm standard error of the mean (SEM). Statistical comparisons were performed using one-way analysis of variance (ANOVA) and the Newman-Keuls test. $\mathrm{P}<0.05$ was indicated a statistically significant difference.

\section{Results}

Changes of ventricular hemodynamic parameters. LVDP, HR and RPP in the DM groups were significantly decreased compared to the control group (Fig. 1). LVDP, HR and RPP were also statistically significant among the different stages of DM (Fig. 1). 
Table I. Changes in plasma IL-1, IL-4 and cardiac 4-HNE levels in the different groups of rats.

\begin{tabular}{lrrrr}
\hline & \multicolumn{3}{c}{ Groups } \\
\cline { 2 - 5 } Variable & \multicolumn{1}{c}{ Control } & \multicolumn{1}{c}{ DM4w } & DM8w & DM12w \\
\hline IL-1 $(\mathrm{pg} / \mathrm{ml})$ & $29.60 \pm 9.64$ & $99.22 \pm 24.04^{\mathrm{a}}$ & $249.03 \pm 36.17^{\mathrm{a}, \mathrm{c}}$ & $437.73 \pm 67.08^{\mathrm{a}, \mathrm{c}, \mathrm{d}}$ \\
IL-4 $(\mathrm{pg} / \mathrm{ml})$ & $42.20 \pm 6.28$ & $31.1 \pm 5.27^{\mathrm{a}}$ & $24.2 \pm 4.39^{\mathrm{a}, \mathrm{b}}$ & $14.2 \pm 5.10^{\mathrm{a}, \mathrm{c}, \mathrm{d}}$ \\
$4-\mathrm{HNE}(\mathrm{ng} / \mathrm{l})$ & $5.65 \pm 0.51$ & $28.16 \pm 7.73^{\mathrm{a}}$ & $45.16 \pm 11.2^{\mathrm{a}, \mathrm{c}}$ & $57.80 \pm 6.06^{\mathrm{a}, \mathrm{c}, \mathrm{d}}$ \\
\hline
\end{tabular}

${ }^{a} \mathrm{P}<0.01$ compared to the control group; ${ }^{b} \mathrm{P}<0.05$, ${ }^{\mathrm{C}} \mathrm{P}<0.01$ compared to the $\mathrm{DM} 4 \mathrm{w}$ group; ${ }^{\mathrm{d}} \mathrm{P}<0.01$ compared to the DM8w group. IL- 1 , interleukin-1; IL-2, interleukin-2; 4-HNE, 4-hydroxynon-2-enal.

A

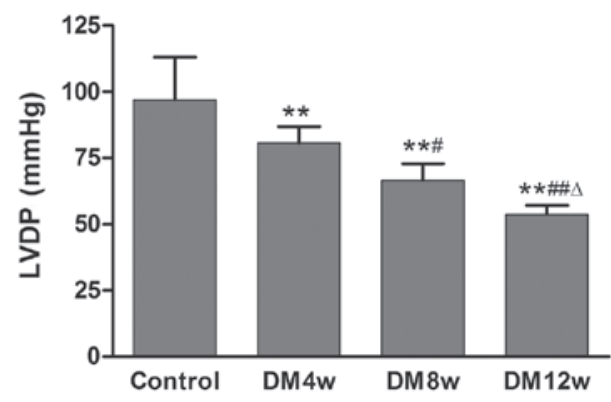

B

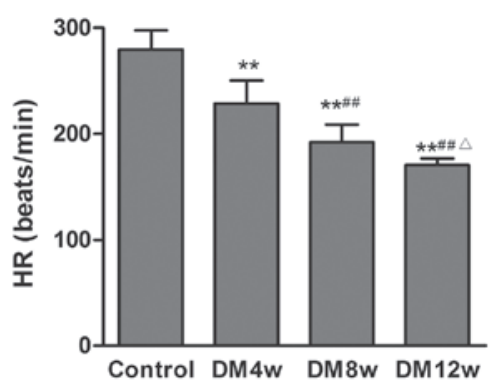

C

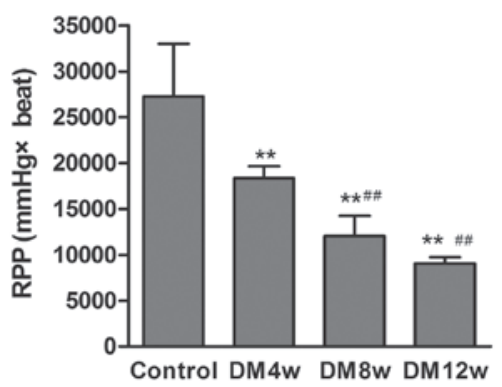

Figure 1. Changes of (A) the left ventricular developed pressure (LVDP) (B) heart rate (HR) and (C) rate-pressure product (RPP) in diabetic rats. ${ }^{* *} \mathrm{P}<0.01$ compared to the control group; ${ }^{\#} \mathrm{P}<0.05,{ }^{\# \prime} \mathrm{P}<0.01$ compared to the DM4w group; ${ }^{\wedge} \mathrm{P}<0.05$ compared to the DM8w group.

Changes of FBG and HbAlc levels. FBG levels were significantly increased in the DM groups compared to the control group, while there were no statistically significant differences among the different stages of DM (Fig. 2A). HbAlc levels were significantly increased in the DM4w, DM8w and DM12w groups compared to the control group, and statistically significant differences were observed among the different stages of DM (Fig. 2B).
A

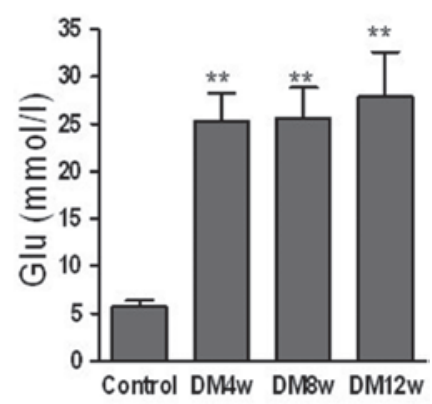

B

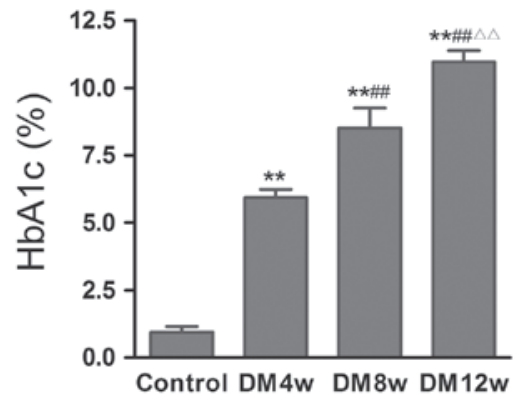

Figure 2. Levels of (A) fasting blood glucose (FBG) and (B) glycosylated hemoglobin $(\mathrm{HbA} 1 \mathrm{c}) .{ }^{* *} \mathrm{P}<0.01$ compared to the control group; ${ }^{\# \#} \mathrm{P}<0.01 \mathrm{com}-$ pared to the DM4w group; ${ }^{\triangle} \mathrm{P}<0.01$ compared to the DM8w group.

Changes of plasma IL-1, IL-4 and cardiac 4-HNE levels. The changes of plasma IL-1, IL-4 and cardiac 4-HNE in different stages of diabetes are shown in Table I. IL-1 and cardiac 4-HNE levels were significantly increased, while IL-4 levels were decreased in the DM groups compared to the control group with progression of diabetes.

Changes of Bax and Bcl-2 mRNA levels in hearts of rat. The changes of Bax and Bcl-2 mRNA levels in the different stages of diabetes are shown in Table II. The mRNA expression levels of cardiac Bax were increased, while Bcl-2 mRNA levels were decreased in the DM groups compared to the control group. The Bcl-2 mRNA levels were also decreased in the different stages of DM. The Bcl-2/Bax mRNA ratios were further decreased with the progression of diabetes (Fig. 3).

Changes of ALDH2 activity in the heart tissue of rats. ALDH2 activity was decreased in the DM groups compared to the control group, with the progression of DM (Fig. 4). 
Table II. Bcl-2 and Bax mRNA expression in the heart tissue of rats.

\begin{tabular}{|c|c|c|c|c|}
\hline \multirow[b]{2}{*}{ Variable } & \multicolumn{4}{|c|}{ Groups } \\
\hline & Control & DM4w & DM8w & DM12w \\
\hline $\operatorname{Bax} / \beta$-actin & $0.55 \pm 0.05$ & $0.67 \pm 0.09^{\mathrm{a}}$ & $0.84 \pm 0.06^{\mathrm{a}, \mathrm{b}}$ & $0.92 \pm 0.04^{\mathrm{a}-\mathrm{c}}$ \\
\hline $\mathrm{Bcl}-2 / \beta$-actin & $0.75 \pm 0.09$ & $0.66 \pm 0.04^{\mathrm{a}}$ & $0.49 \pm 0.03^{\mathrm{a}, \mathrm{b}}$ & $0.37 \pm 0.04^{\mathrm{a}-\mathrm{c}}$ \\
\hline $\mathrm{Bcl}-2 / \mathrm{Bax}$ & $1.38 \pm 0.26$ & $1.02 \pm 0.13^{\mathrm{a}}$ & $0.58 \pm 0.05^{\mathrm{a}, \mathrm{b}}$ & $0.40 \pm 0.03^{\mathrm{a}-\mathrm{c}}$ \\
\hline
\end{tabular}

${ }^{\mathrm{a}} \mathrm{P}<0.01$ compared to the control group; ${ }^{\mathrm{b}} \mathrm{P}<0.01$ compared to the $\mathrm{DM} 4 \mathrm{w}$ group; ${ }^{\mathrm{c}} \mathrm{P}<0.05$ compared to the DM8w group.

A
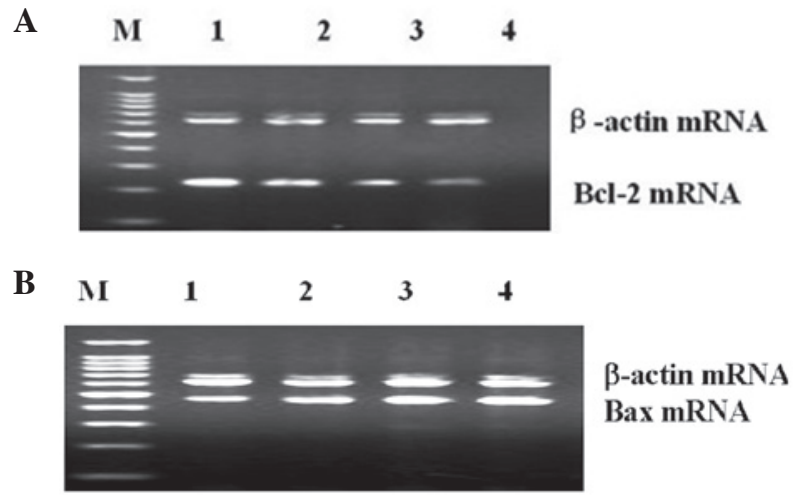

Figure 3. Cardiac (A) Bcl-2 and (B) Bax mRNA expression in control and DM rats. M, marker; lanes: 1, control group; 2, DM4w group; 3, DM8w group; 4, DM12w group.

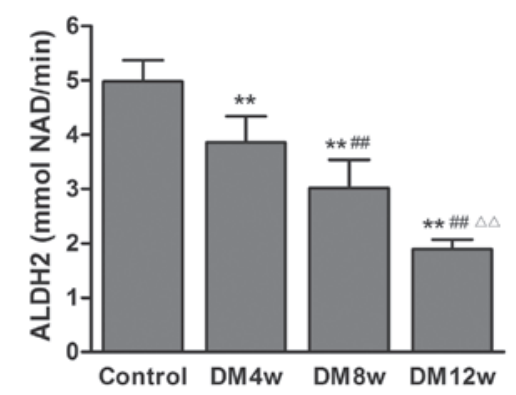

Figure 4. Changes of cardiac aldehyde dehydrogenase 2 (ALDH2) activity in control and DM rats. ${ }^{* *} \mathrm{P}<0.01$ compared to the control group; ${ }^{\# \#} \mathrm{P}<0.01$ compared to the DM4w group; ${ }^{\triangle} \triangle \mathrm{P}<0.01$ compared to the DM8w group.

\section{Discussion}

In the present study, FBG and HbAlc levels were found to be increased, ventricular function was decreased, plasma IL-1 levels were increased, while IL-4 levels were decreased with progression of DM. Cardiac 4-HNE levels were increased and ALDH2 activity was decreased, suggesting that inflammatory injury and oxidative stress were aggravated in diabetes accompanied by a decrease in ventricular function, which may be related to the decrease in ALDH2 activity.

Inflammation plays a key role in inflammatory diseases including diabetes, heart disease, Alzheimer's and Parkinson's disease. Inflammation is the activation of the immune system in response to infection, injury or irritation. ROS, pro- and anti-inflammatory cytokines are often involved in the pathogenesis of inflammation. ROS trigger a cascade of events, including the upregulation of pro-inflammatory cytokines that activate immune responses and determine occurrence of inflammation (20). Pro-inflammatory cytokines, such as IL-1, IL-2 and interferon (IFN)- $\gamma$, are secreted by Th1 cells. Th1 subsets promote cell immune response, cause the release of pro-inflammatory cytokines, and subsequently induce an increase in free radical production and the destruction of $\beta$-cells (21). However, anti-inflammatory cytokines, such as IL-4, are also secreted by Th 2 cells. The proliferation and differentiation of $\beta$-cells is stimulated by IL- 4 , which also promotes the growth of thymus cells with pro-activated $\mathrm{T}$ cells, inhibits the precursor of the helper T cell (Th-p) shift to Th1 cells, and induces the inhibition of monocyte/macrophage cells to produce INF- $\alpha$ and IL-1. IL-4 is important in immune regulation and inflammatory injury (22). In the present study, the results showed that plasma IL-1 levels were increased and that IL-4 levels were decreased in DM rats, suggesting that inflammtory injury was aggravated with the progression of diabetes.

4-HNE is the main product of oxidative stress. Under pathological conditions, these detoxification pathways fail to trigger the accumulation of oxidized lipids that damage key proteins in the mitochondrial respiratory chain. 4-HNE reacts with protein at the imidazole(s) of histidine, the sulfhydryl group of cysteine, and/or the $\varepsilon$-amine of lysine and induces enzyme inactivation (23). Therefore, the generation of lipid peroxidation products capable of reacting with cellular nucleophiles may be primary instigators of tissue injury (24). In the present study, we observed that cardiac 4-HNE levels were increased with the progression of diabetes, suggesting that oxidative stress was aggravated in the hearts of DM rats.

$\mathrm{ALDH} 2$ is a tetrameric enzyme that is present in organs which require high mitochondrial capacity of oxidative ATP generation, such as the heart and brain. In addition to its dehydrogenase activity, ALDH2 functions as an esterase and reductase, depending on the substrates. ALDH2 activation facilitates the removal of cytotoxic aldehydes, such as 4-HNE, reduces myocardial injury, and ultimately is involved in anti-oxidative stress. It has been reported that ALDH2 protects against ethanol toxicity through altered Akt and AMPK signaling pathways, increases its enzymatic activity, diminishes the pro-apoptotic signaling activity of JNK $1 / 2$ and reduces 4-HNE protein adduct formation $(25,26)$. In the present 
study, 4-HNE content was increased when ALDH2 activity was decreased with the progression of diabetes, suggesting that the decrease in ALDH2 activity leads to the metabolic enhancement of acetaldehyde activity and the aggravation of cardiac injury.

Apoptosis occurs in diabetic cardiomyopathy. It is believed that the ultimate vulnerability of cells to various apoptotic stimuli is determined by the relative ratio of pro-apoptotic and anti-apoptotic members of the Bcl-2 family. Accumulating evidence has proven that the overproduction of ROS triggers myocyte apoptosis by upregulating pro-apoptotic members of the Bcl-2 family, such as Bax, Bak and Bid. Bax plays a dominant role in initiating cell death, thus disrupting the integrity of mitochondrial membrane, and hastening the release of cytochrome $c$ from mitochondria, leading to DNA fragmentation and caspase- 3 activation. However, Bcl-2 exerts an opposite effect. In the present study, Bax and Bcl-2 mRNA levels were detected. The result showed that the mRNA expression of cardiac Bcl-2 was decreased, Bax at mRNA levels was increased, and $\mathrm{Bcl}-2 / \mathrm{Bax}$ mRNA ratios were decreased with progression of DM. ALDH2 activity was also found to be decreased. These results suggest that the increased apoptosis of myocardial cells is associated with the decrease in ALDH2 activity.

In conclusion, with the development of diabetes, which is accompanied by the decrease of cardiac ALDH2 activity, inflammatory injury, oxidative stress and apoptosis were aggravated in the hearts of DM rats. This finding suggests that ALDH2 is an important regulator of diabetic cardiomyopathy. Additional studies are needed to confirm this potential role of ALDH2.

\section{Acknowledgements}

This study was supported by research grants from the National Natural Science Foundation of China (nos. 81000074 and 81170046) and the Anhui Province Natural Science Foundation of China (no. 1208085MH131).

\section{References}

1. Adeghate E, Schattner P and Dunn E: An update on the etiology and epidemiology of diabetes mellitus. Ann N Y Acad Sci 1084: 1-29, 2006.

2. Gremizzi C, Vergani A, Paloschi V and Secchi A: Impact of pancreas transplantation on type 1 diabetes-related complications. Curr Opin Organ Transplant 15: 119-123, 2010.

3. Boudina S and Abel ED: Diabetic cardiomyopathy revisited. Circulation 115: 3213-3223, 2007.

4. Zgibor JC, Ruppert K, Orchard TJ, Soedamah-Muthu SS, Fuller J, Chaturvedi N and Roberts MS: Development of a coronary heart disease risk prediction model for type 1 diabetes: the Pittsburgh CHD in Type 1 diabetes risk model. Diabetes Res Clin Pract 88: 314-321, 2010.

5. Kaneto H, Katakami N, Matsuhisa M and Matsuoka TA: Role of reactive oxygen species in the progression of type 2 diabetes and atherosclerosis. Mediators Inflamm 2010: 453892, 2010.

6. Penckofer S, Schwertz D and Florczak K: Oxidative stress and cardiovascular disease in type 2 diabetes: the role of antioxidants and pro-oxidants. J Cardiovasc Nurs 16: 68-85, 2002.
7. Ren J: Acetaldehyde and alcoholic cardiomyopathy: lessons from the ADH and ALDH2 transgenic models. Novartis Found Symp 285: 69-79, 2007.

8. Budas GR, Disatnik MH and Mochly-Rosen D: Aldehyde dehydrogenase 2 in cardiac protection: a new therapeutic target? Trends Cardiovasc Med 19: 158-164, 2009.

9. Forman HJ, Fukuto JM, Miller T, Zhang H, Rinna A and Levy S: The chemistry of cell signaling by reactive oxygen and nitrogen species and 4-hydroxynonenal. Arch Biochem Biophys 477: 183-195, 2008.

10. Petersen DR and Doorn JA: Reactions of 4-hydroxynonenal with proteins and cellular targets. Free Radic Biol Med 37: 937-945, 2004.

11. Ma H, Li J, Gao F and Ren J: Aldehyde dehydrogenase 2 ameliorates acute cardiac toxicity of ethanol: role of protein phosphatase and forkhead transcription factor. J Am Coll Cardiol 54: 2187-2196, 2009.

12. Doser TA, Turdi S, Thomasm DP, Epstein PN, Li SY and Ren J: Transgenic overexpression of aldehyde dehydrogenase-2 rescues chronic alcohol intake-induced myocardial hypertrophy and contractile dysfunction. Circulation 119: 1941-1949, 2009.

13. Ge W, Guo R and Ren J: AMP-dependent kinase and autophagic flux are involved in aldehyde dehydrogenase-2-induced protection against cardiac toxicity of ethanol. Free Radic Biol Med 51: 1736-1748, 2011.

14. Churchill EN, Disatnik MH and Mochly-Rosen D: Time-dependent and ethanol-induced cardiac protection from ischemia mediated by mitochondrial translocation of varepsilonPKC and activation of aldehyde dehydrogenase 2. J Mol Cell Cardiol 46: 278-284, 2009.

15. Chen CH, Budas GR, Churchill EN, Disatnik MH, Hurley TD and Mochly-Rosen D: Activation of aldehyde dehydrogenase-2 reduces ischemic damage to the heart. Science 321: 1493-1495, 2008.

16. Budas GR, Disatnik MH, Chen $\mathrm{CH}$ and Mochly-Rosen D: Activation of aldehyde dehydrogenase 2 (ALDH2) confers cardioprotection in protein kinase $\mathrm{C}$ epsilon (PKCvarepsilon) knockout mice. J Mol Cell Cardiol 48: 757-764, 2010.

17. Wang J, Wang H, Hao P, Xue P, Wei S, Zhang Y and Chen Y: Inhibition of aldehyde dehydrogenase 2 by oxidative stress is associated with cardiac dysfunction in diabetic rats. Mol Med 17: 172-179, 2011.

18. Larsen CM, Faulenbach M, Vaag A, Ehses JA, Donath MY and Mandrup-Poulsen T: Sustained effects of interleukin-1 receptor antagonist treatment in type 2 diabetes. Diabetes Care 32: 1663-1668, 2009.

19. Zaitsev SV, Appelskog IB, Kapelioukh IL, Yang SN, Köhler M, Efendic S and Berggren PO: Imidazoline compounds protect against interleukin 1 beta-induced beta-cell apoptosis. Diabetes 50: 70-76, 2001 .

20. Tas SW, Remans PH, Reedguist KA and Tak PP: Signal transduction pathways and transcription factors as therapeutic targets in inflammatory disease: towards innovative antirheumatic therapy. Curr Pharm Des 11: 581-611, 2005,

21. Lafaille JJ: The role of helper $\mathrm{T}$ cell subsets in autoimmune diseases. Cytokine Growth Factor Rev 9: 139-151, 1998.

22. Hart PH, Jones CA and Finlay-Jones JJ: Interleukin-4 suppression of monocyte tumour necrosis factor-alpha production. Dependence on protein synthesis but not on cyclic AMP production. Immunology 76: 560-565, 1992.

23. Zhang $M$ and Shah AM: Role of reactive oxygen species in myocardial remodeling. Curr Heart Fail Rep 4: 26-30, 2007.

24. Lee Y and Gustafsson AB: Role of apoptosis in cardiovascular disease. Apoptosis 14: 536-548, 2009.

25. Stewart MJ, Malek K and Crabb DW: Distribution of messenger RNAs for aldehyde dehydrogenase 1, aldehyde dehydrogenase 2, and aldehyde dehydrogenase 5 in human tissues. J Investig Med 44: 42-46, 1996.

26. Esterbauer H, Schaur RJ and Zollner H: Chemistry and biochemistry of 4-hydroxynonenal, malonaldehyde and related aldehydes. Free Radic Biol Med 1: 81-128, 1991. 